\title{
Las Redes Comunitarias y Familiares en el tránsito hacia la externación sustentable en el contexto de la reforma del Modelo de Atención de Salud Mental en Uruguay

\author{
Community and Family Networks in the transition towards sustainable \\ externalization in the context of the reform of the Mental Health Care \\ Model in Uruguay
} \\ Redes Comunitárias e Familiares na transição para a externalização sustentável no contexto da reforma do Modelo de Atenção à Saúde Mental no Uruguai
}

Claudia Morosi ${ }^{1}$, Álvaro Díaz ${ }^{2}$, Laura Martínez ${ }^{3}$

\section{Resumen:}

El presente artículo toma como eje de discusión el lugar de las redes comunitarias y familiares en la estrategia de desinstitucionalización. La propuesta se plantea en el contexto de reforma del modelo de atención de salud mental del Sistema Nacional integrado de Salud (SNIS) que se viene dando en Uruguay a partir de la Ley Nro. 19529 de Salud Mental del año 2017, para pensar las prácticas respecto al modelo de atención de salud mental comunitaria. Esto implica dar visibilidad a las condiciones materiales de existencia en la cotidianeidad de las personas, al bienestar individual y colectivo, al trabajo, la vivienda, la educación, la cultura, al tiempo de ocio y disfrute, entre otros. La transición del modelo asilar a un modelo que privilegie el enfoque comunitario debe considerar en primer lugar un cambio de la política pública de salud mental que contemple los distintos actores, entre ellos los usuarios, familiares, trabajadores de la salud y la comunidad en general. La reinserción en el tejido social de las personas hospitalizadas es central a la hora de pensar en una externación sustentable, por lo que efectivizar la inclusión social va a significar cambios en las

\footnotetext{
${ }^{1}$ Licenciada en Sociología (UdelaR) Prof adj. Dto. de Enfermería Comunitaria de la Facultad de Enfermería de la UdelaR. Prof. adj. de la Facultad de Medicina de la UdelaR. Magister en bioética (ULIA)- Doctoranda en Ciencias de la Salud (UfpeL). ORCID: 0000-0002-4239-5106 correo electrónico: claudiamorosi@gmail.com ${ }^{2}$ Licenciado en Enfermería (UdelaR) Prof. Agdo. Departamento de Enfermería en Salud Mental de la Facultad de Enfermería de la UdelaR. Especialista en salud mental y salud Familiar y Comunitaria. Magister en Salud Mental. Facultad de Enfermería (UdelaR) Dr. En Ciencias de la Salud.(UfpeL) ORCID: 0000-00033382-6004 correo electrónico: adiaz90@hotmail.com

${ }^{3}$ Licenciada en Psicología (UdelaR). Especialista en Intervenciones Comunitarias en Salud (UdelaR). Integrante del Dpto. de Psicología de Hospital Vilardebó. Diplomada en Política de Drogas, Regulación y Control (UdelaR). ORCID: 0000-0002-4042-3986 correo electrónico: lauravemc@gmail.com
} 
prácticas sanitarias en los recursos de salud que permitan el sostén familiar y comunitario, para que las personas en situación de sufrimiento psíquico puedan encontrar oportunidades de desarrollar la vida por fuera del hospital psiquiátrico, siendo respetadas en sus derechos.

\section{Palabras Clave:}

Modelos de atención en salud mental, desinstitucionalización, salud mental.

\section{Abstract:}

This article takes as a main topic of discussion the place community and family networks take at the strategy of deinstitutionalization. The project is presented in the context of the Mental Healthcare System of the national integrated health system (SNIS) reform that has been taking place in Uruguay from the Law No. 19.529 of Mental Health (2017) to think about the practices regarding the Mental Healthcare Community Model. This implies giving visibility to the material conditions of existence in people's daily lives, to individual and collective well-being, to work, housing, education, culture, leisure and enjoyment time, among others. The transition from an asylum model to a model that privileges the community approach, must first consider a change in the public mental health policy that contemplates different actors, including users, their relatives, health workers and the community in general. The reintegration into the social fabric of hospitalized people is central when it comes to thinking about a sustainable discharge, and that is why making social inclusion effective will imply changes in the sanitarian practices, in health resources, which allow family and community support, so that those in psychological suffering can find opportunities to develop their lives outside the psychiatric hospital, being respected in their rights

\section{Keywords:}

Mental Healthcare Models, Deinstitutionalization, Mental Healt.

\section{Resumo:}

O presente artigo toma como eixo de discussão o lugar das redes comunitárias e familiares na estratégia de desinstitucionalização. A proposta é apresentada no contexto da reforma do modelo de atenção do sistema de saúde mentaldo sistema nacional integrado de saúde (SNIS) que vem ocorrendo no Uruguai a partir da Lei no 19529 de Saúde Mental de 2017 para pensar as práticas referentes ao modelo de atenção à saúde comunidade mental. Isso implica dar visibilidade às condições materiais de existência no cotidiano das pessoas, ao bem-estar individual e coletivo, ao trabalho, moradia, educação, cultura, lazer e prazer, entre outros. A transição do modelo de asilo para um modelo que privilegia a abordagem da comunidade deve primeiro considerar uma mudança na política pública de saúde mental que inclua diferentes atores, incluindo usuários, familiares, trabalhadores de saúde e a comunidade em geral. A reintegração no tecido social das pessoas hospitali- 
zadas é central quando se trata de pensar uma externalização sustentável, para que a inclusão social efetiva signifique mudanças nas práticas de saúde, nos recursos de saúde, que permitam o apoio familiar e comunidade, para que as pessoas em situação de sofrimento psíquico encontrem oportunidades de desenvolver a vida fora do hospital psiquiátrico, sendo respeitadas em seus direitos.

\section{Palavras-chave:}

Modelos de atenção em saúde mental, desinstitucionalização, saúde mental.

\section{Introducción}

La propuesta de investigación surge de la demanda concreta que establece el Departamento de Trabajo Social del Hospital Vilardebó. La misma, consiste en visualizar las formas institucionales que han obstaculizado y/o posibilitado el alta hospitalaria con el objetivo de avanzar en lo que el equipo investigador define como externación sustentable, así como, comenzar un proceso de problematización sobre el trabajo profesional que intente conocer los contenidos simbólicos y subjetivos que se materializan en esas prácticas y acompañe la coyuntura actual de plena discusión acerca de la puesta en vigencia de la Ley de salud mental.

Para proporcionar mejor visibilidad de las prácticas profesionales el equipo de investigación realizo entrevistas semi-estructuradas a profesionales que desempeñan funciones en salas abiertas en el Hospital Teodoro Vilardebó, con el aval Institucional de ASSE y la correspondiente aprobación del Comité de Ética de la Institución con fecha marzo de 2017. Los profesionales entrevistados fueron: tres Licenciados en Enfermería, seis de Trabajo Social, cinco de Psicología y seis Psiquiatras desde dos perspectivas que permitan el análisis de las lógicas manicomiales; la primera en términos de ideal, como aquello que debe ser y la segunda en términos concretos. Esta diferenciación fue fundamental para dar sentido a la palabra, y en términos concretos comprenderla práctica cotidiana.

En el campo de la salud mental la obsolescencia del modelo asilar, para dar tratamiento a las personas en situación de sufrimiento mental, nos interpela sobre las posibilidades reales que tiene las personas una vez fuera del hospital, para efectivizar en la comunidad el ejercicio de sus derechos en el goce de una vida digna que enmiende los faltas de las lógicas manicomiales. Entendemos que la escisión entre lo hospitalario y el contexto social que conforma la vida de las personas devenidas "pacientes psiquiátricos" no aporta a la comprensión exhaustiva de las múltiples dimensiones que atraviesan las personas en los procesos de institucionalización psiquiátrica. Es en este sentido que se busca problematizar el concepto de redes comunitarias, familiares y sociales con el fin de avanzar en la des-psiquiatrización de la producción de conocimiento en el campo de la salud mental.

Partimos de la premisa de que la conexión entre el hospital y las redes comunitarias y familiares son fundamentales a la hora de concretar el egreso de la persona, y el lugar social del "pa- 
ciente psiquiátrico", identificando barreras en la generación de redes que propicien alternativas al circuito psiquiátrico.

La investigación pone énfasis en una perspectiva de derechos humanos desde una concepción integral, inspirados en el reconocimiento, respeto y promoción de los mismos, y desde esta integralidad el espacio biopolítico moderno nos permite analizar uno de los campos sociales más discutidos en los últimos dos años en nuestro país como es el de la salud mental.

\section{Fortalezas y obstáculos para la externación sustentable.}

El Equipo de investigación definió "externación sustentable" como la salida hospitalaria con el consecuente proceso externo al psiquiátrico, de desarrollo de la vida personal en sus diversas dimensiones y de permanente tensión en la sostenibilidad de esa situación de externalidad frente a las barreras del medio y la potencialidad de la persona. Este concepto comprende el de "alta médica" a la vez que lo complejiza, ya que requiere necesariamente del abordaje interdisciplinario para la proyección y la efectivización de la externación y también de su sostenimiento, traducido en procesos de inclusión genuinos.

Algunas de las frases que se reiteran en los discursos de los profesionales entrevistados para la investigación están relacionadas con los obstáculos y fortalezas para la externación hacen referencia a que:

"Es importante trabajar de manera interdisciplinaria para concretar la salida"

"Tender a modelos menos hospitalocéntricos".

"La des-hospitalización debe hacerse por grupo"
"Son importantes los trabajos intersectoriales."

"No tener casas de medio camino"

"En las Policlínicas periféricas no se hace atención primaria ni prevención sino asistencia."

"El trabajo de Policlínica es una mochila"

"Carencias en los recursos en general y carencias de recursos para determinados perfiles de usuarios."

Hablar de externación sustentable admite una configuración de trabajo en salud mental comunitaria desde una perspectiva de derechos que nos permita pensar en procesos de reforma de la lógica del modelo asilar, patologizante y vulnerador de derechos que ha reforzado el estigma y alejado a los individuos de su entorno y comunidad. Desde esta perspectiva parecería inminente que la situación de sufrimiento psíquico es siempre vista desde el lugar de la otredad extrema; de allí el concepto de monstruosidad que plantea Foucault en su texto de "Los anormales". Es justamente esta concepción hegemónica de la salud mental, la que debemos cuestionar en su calidad establecida como "absoluta", con el fin de promover líneas que habiliten un verdadero cambio en el modelo de atención. La externación sustentable exige pensar en el fortalecimiento de las redes socio-comunitarias y familiares que permitan sostener en su cotidianeidad a las personas que se encuentran en situación de sufrimiento psíquico. Estas redes establecen escenarios locales que incentivan intercambios interpersonales, realzan las conductas preventivas y promueven prácticas horizontales de trasmisión de conocimiento. 


\section{El entramado de redes de apoyo comunitarias y familiares}

La metáfora de la sociedad como tejido refiere al entrelazamiento de las vidas y acciones de las personas que la conforman, entendido como un entramado de redes múltiples que van a permitir analizar al sujeto y su contexto. Este entramado de redes no es único sino múltiple y permite entender la presencia de lo social en el individuo, la vida con el "otro" y la función de ese "otro" en su subjetividad, y su contexto en términos de malla social, económica, política y cultural que sostiene y amortigua los vaivenes de la vida en la sociedad capitalista, poniendo al descubierto la complejidad de las dinámicas vinculares. La vida en común tiene, además del aspecto individual para establecer relaciones con los otros, el componente que Bourdieu llamó "capital social”, determinado por el lugar de nacimiento a través de la familia, el lugar que ocupa en la jerarquía social, acceso a la educación, empleo. Ambos aspectos nos permiten determinar el grado de integración social del individuo.

El concepto de "apoyo social " surgió al revisar en los años 70 la literatura que parecía evidenciar una asociación entre problemas psiquiátricos y variables sociales genéricas como la desintegración social, movilidad geográfica etc.

Según Quesada, Kessler ${ }^{(1)}$, en 1985 detectó:

...que el elemento común de esas variables situacionales era la ausencia de lazos sociales adecuados o la ruptura de redes sociales previamente existentes. Desde entonces existe una importante línea de investigación en apoyo social y la mayor parte de los autores identifican de una u otra forma el apoyo social con la relación interpersonal en la que se ofrece o intercambia ayuda de tipo material, instrumental o emocional que produce sensación de bienestar al receptor.

La intervención comunitaria adquiere sentido si incorporamos la importancia que el apoyo social tiene en la salud y el bienestar, las transiciones vitales y las situaciones de crisis y se desarrollan trabajos en redes desde un punto de vista individual - familiar y comunitario en una determinada población. Para eso se deben elaborar estrategias concretas que potencien y complementen recursos existentes.

$\mathrm{El}$ apoyo funciona a nivel individual y social, y está comprobado que las personas se sienten cuidadas cuando pertenecen a una red social. La cantidad de apoyo social y emocional que recibe la gente varía según su estatus social y económico, por lo que la pobreza puede contribuir al aislamiento y a la marginación social. Diseñar instalaciones donde se fomenten los encuentros y la interacción social en el afuera puede ayudar a mejorar la salud mental; por lo tanto visibilizar y activar las redes para sostener en el afuera significa tener una perspectiva de red donde tengamos conocimiento de la totalidad del contexto del individuo, realizando el trabajo de manera interdisciplinaria, involucrando a todos los actores (sanitarios, familiares, comunitarios, etc.).

La externación es un proceso muy sensible en salud mental, ya que pone en juego procedimientos interdisciplinarios complejos que van desde, dar seguimiento a la salud en la atención de la persona, garantizar la inserción en su comunidad hasta la promoción de derechos, lo que implica tener en cuenta recursos humanos y materiales, medidas y estrategias para asegurar la sustentabilidad. Este, en el modelo 
médico hegemónico tradicional, se limita a dar la indicación al paciente y/o allegados para que retorne a la consulta, a veces periódicamente, y siempre dentro de espacios institucionales formales. Los otros componentes informales y sociales no son tenidos en cuenta ni integrados. La importancia de las redes comunitarias y familiares se ha empezado a visibilizar de manera muy reciente debido a la importancia e incidencia en los procesos dialecticos de salud-enfermedad. De este modo, la consideración de las redes sociales y el trabajo con ellas se han vuelto imprescindibles en las prácticas sanitarias a todo nivel, sobre todo en el fenómeno-proceso de la externación, y particularmente en salud mental. Las redes propician el vínculo entre el usuario y el profesional de la salud, por su trato más estrecho y constante no sólo con "el paciente" sino con su familia y comunidad.

Si analizamos el articulado de la ley de Salud Mental Nro. 19529 que hacen mención a las redes, vemos que en el capítulo $\mathrm{V}$ se pone énfasis en los niveles de complejidad que tendrá la atención a la salud mental destacándose como estrategia la APS (Atención Primaria en Salud) y priorizando el primer nivel de atención.

En su artículo $17^{(2)}$ la Ley de Salud Mental destaca: "El proceso de atención debe realizarse preferentemente en el ámbito comunitario (...) Esta atención se realizará en el marco de un abordaje interdisciplinario e intersectorial y estará orientado a la promoción, reforzamiento y restitución de los lazos sociales."

La ley busca una reforma del sistema de asistencia a la Salud Mental y nos preguntamos ¿cómo se efectivizará la promoción, reforzamiento y restitución de los lazos sociales que conforman este tejido cuando hemos venido asistiendo a una lógica donde el afuera no sostiene a las personas con sufrimiento mental?
A su vez el artículo 18 la Ley ${ }^{(2)}$ de Salud Mental señala: "Se fomentará el establecimiento de redes territoriales de atención, las que a su vez podrán articular su labor en cada zona con otros recursos existentes que puedan aportar a la promoción y prevención en salud mental."

Concebimos las redes comunitarias como Montero (3) "como parte de la actividad y resistencia de las comunidades, de su expresión como sociedad civil, a la vez que muestran su capacidad de transformación y de apoyo social, su poder, su carácter fortalecedor..."

Nos preguntamos ¿cómo se fomentará el establecimiento de redes territoriales que contribuyan a revertir el aislamiento y la marginación social? Sobre todo cuando se sabe que el apoyo funciona a nivel individual y social y que las personas se sienten cuidadas cuando pertenecen a una red social, y que en situaciones de vulnerabilidad el fortalecer redes sociales tanto a nivel comunitario como familiar permiten sostener al sujeto.

Cualquier forma de atención en salud deberá tener en cuenta las posibles redes sociales de cada persona, particularmente en lo que hace a la externación y para que esta se vuelva sustentable. Ello implica en primer término visualizar los distintos vínculos y grupos que pueden hacer de red para cada persona, y trabajar con los mismos en forma interdisciplinaria por parte de los distintos profesionales de la salud involucrados: enfermeros, trabajadores sociales, sociólogos, psicólogos, médicos, odontólogos.

Este equipo deberá visualizar la red como primer paso, luego contactar a alguno o algunos de sus integrantes y reafirmar en ellos la conciencia de su importancia como grupo humano de apoyo imprescindible para la persona; también mantendrá un contacto constante con estas per- 
sonas, así como con los sectores e instituciones de su comunidad: escuela, trabajo, municipio, clubes y centros sociales. Se trata así de reforzar la red ya existente y coordinar con ella acciones y medidas para el seguimiento de la o las personas en cuestión y la consolidación plena de su externación.

Algunas de las expresiones recurrentes entre los distintos profesionales, vinculadas a las redes comunitarias hacen referencia a que:

"Es fundamental la conexión entre el hospital y la red comunitaria para lograr proyectos de egreso con el paciente."

"Se necesitan estructuras chicas que sostengan más."

"Desconocimiento de las redes familiares y comunitarias "

"No se comparten las visiones del proceso salud-enfermedad con las redes."

"La mayoría de los profesionales desconocen las interconexiones."

"Las redes no son homogéneas ni democráticas en el acceso."

"El hecho de que las redes comunitarias a veces no estén presentes por fallas en las funciones de la gestión institucional y de los profesionales"

El trabajo en redes desde la mirada de los profesionales de la salud implica no solo salir del servicio desde un programa de cercanía, recorrer el barrio, sino intercambiar desde otra lógica, trabajar en la vida cotidiana de una comunidad. Esta forma de abordaje nos permite una perspectiva ética-política de abordaje profesional muy útil en el encuentro con un otro.

Es por ello que lo que aporta sentido al paradigma de atención en salud mental comunitaria es "el con quién hago el proceso" y no para quién. El abordaje está significado como acción social y por lo tanto conlleva la idea de la paridad entre los equipos profesionales y las personas. Éste encuentro es elemento primordial para la producción de cualquier cambio basado en el vínculo y las relaciones.

El entramado de redes hace que sean varios los agentes socializadores que aborden al individuo poniendo al descubierto la complejidad de las dinámicas vinculares.

\section{"Personas que no tienen un lugar en el mundo"}

Los discursos coinciden en que los pacientes internados son "pacientes que no tienen un lugar en el mundo" "un afuera que sostenga" interpelando y cuestionando modelos familiares.

Berenstein $^{(4)}$ destaca que cuando un grupo familiar se organiza por medio del eje salud/enfermedad contiene otras participaciones correspondientes a la organización social, económica, religiosa, psicológica.

La organización dualista, a nivel familiar agrupa diversas categorías. Cuando se escinde en enfermos y sanos alcanza una organización duradera, determinada y determinante de la organización familiar y lleva al grupo a buscar en el contexto social el marco para avalar esta organización.

Las personas que no tienen lugar en el mundo, y un afuera que los sostengan ocupan ese lugar en la organización que se ha venido constituyendo no de manera súbita sino como consecuencia de una reorganización sucesiva de explicaciones normativas, poco explicitas y no sistemáticas. La familia sigue los lineamientos de las normas sociales, adaptadas al criterio vigente y desde donde las conductas normales son aquellas que la sociedad considera como tales con arreglo a una norma. 
En relación a las fortalezas y obstáculos referidos al vínculo con las familias los profesionales entrevistados hacen mención a que:

"Es importante la existencia de las redes familiares y comunitarias para sostener"

"Siempre el papel nuestro es llamar a la familia y que la familia sea parte de la decisión que vamos a tomar"

"Las cosas cambian cuando se trabaja con la familia."

"La Familia es fundamental, pero la malla familiar esta desgastada y castigada”.

"Familia patologizante."

"No predomina la familia"

"La familia no entiende a veces la enfermedad mental"

En la relación terapéutica el respeto es previo y antecede a la razón. "La moral cobra voz cuando y donde los imperativos de la razón callan o cuando y donde se le niega la voz: cuando y donde la decisión de responsabilizarse del bienestar, la autonomía, la integridad y la subjetividad de otro deja en suspenso la legitimidad y la autoridad de los juicios de la razón y priva a esta de sus credenciales como tribunal de apelación, es decir, como autoridad a la que podemos recurrir para cuestionar las acciones propiciadas por un impulso moral Bauman ${ }^{(5)}$ ".

Diversos enfoques del paradigma de salud mental comunitaria señalan que las causas que originan mayores limitaciones y peores consecuencias en la vida de las personas con sufrimiento mental no son aquellas individuales sino las de construcción y opresión social, entendiendo lo social como constitutivo del individuo y no en cómo el individuo se relaciona con lo social; como se pone de manifiesto en las distintas entrevistas.
De allí la insistencia e importancia de pensar al "otro" y pensarse con el "otro" en términos de una alteridad irreductible como lo denomina Lévinas $^{(6)}$, desde diferentes perspectivas, lo que implica pensar por fuera del sentido común socialmente establecido.

La "otredad" se establece siempre en función de la valoración y el respeto de lo que es diferente a mí aun cuando ese "otro" no encaja en lo que "yo" deseo que sea. En este sentido, corresponde responsabilizarnos y crear acciones que den visibilidad a la multicausalidad de las problemáticas que rodean a las personas con sufrimiento psíquico, que a la vez contribuya a desarrollar un proyecto colectivo comunitario en salud mental que contenga la participación de la comunidad y tenga presente todos los atravesamientos en los procesos que la componen.

El autoanálisis consiste en el trabajo de investigación y de comprensión que las comunidades mismas, como protagonistas de sus necesidades, deseos, intereses y demandas, potencialidades y limites, pueden hacer, en forma continuada, para re adquirir, pero también para inventar un pensamiento y conocimientos, producidos con su propio método y con su propio léxico. Baremblit ${ }^{(7)}$. Respecto a esto es interesante pensar en procesos que permiten re-significar lo establecido, pensar en otras formas que promuevan salud en la sociedad. Pensar los desafíos, interpelar saberes disciplinares que posibilite el encuentro entre servicios y comunidad en una interrelación mutua que permita salirse de los estándares actuales para crecer, trascender lo ya conocido para el cumplimiento de los derechos en salud mental, en el ejercicio de una vida digna. Teniendo en cuenta estos aspectos es que Hopenhayn $^{(8)}$, expresa que: 
La construcción socio-histórica-cultural-ambiental y espiritual de la comunidad en la que nos desarrollamos es relevante en nuestro proceso personal en la medida en que entre otros aspectos implica el desarrollo de la identidad del individuo, su capacidad de decisión y "la posibilidad de contar con redes de relaciones que ayuden a desarrollar el proyecto de vida.

Distintos autores plantean los avances en cuanto a la concepción de salud. Ello supone otra mirada epistemológica en relación al sistema devenido en ausencias y profundas fallas históricas, políticas, sociales, económicas, culturales que han dejado por fuera la complejidad que envuelve la reconstrucción de salud- enfermedad en el proceso dinámico y dialéctico. Proceso en el que se involucra la perspectiva psico-social, emocional, de funcionamiento $y$ adaptación personal en la comunidad.

El Artículo I de la Declaración Universal de los Derechos Humanos Unesco $-1948^{(9)}$ establece que "Todos los seres humanos nacen libres e iguales en dignidad y derechos y, dotados como están de razón y conciencia, deben comportarse fraternalmente los unos con los otros". Desde la perspectiva de la ética kantiana se sostiene que la dignidad humana es una cuestión fundamentalmente moral que radica en la autonomía, por lo que todas las personas poseen la misma dignidad. Es a partir de esta filosofía desde donde éste modelo se encuentra íntimamente relacionado, propiciando ciertos valores intrínsecos como la dignidad humana, la igualdad y a libertad, reconociendo su carácter universal y su configuración en el campo de los derechos humanos. Valores que además aspiran a potenciar el respeto por la diferencia y la libertad personal favoreciendo la inclusión social. Elementos habilitadores, que empoderan a las personas y componen redes de contención para sostener proyectos de vida significativos en sus comunidades.

\section{Conclusiones}

Las prácticas profesionales deben basarse en el reconocimiento del "otro" como sujeto de derechos y no en el positivismo de Comte, tan sustentado desde la psiquiatría en el sentido del paciente como un "objeto natural."

A partir de la investigación y del análisis de las entrevistas se percibe la importancia de la perspectiva comunitaria y familiar en el discurso de salud mental y en prácticas que den visibilidad al "otro".

La ausencia de lazos sociales y/o la ruptura de redes familiares o comunitarias existentes ha sido un elemento común cuando se analizan variables sociales genéricas como la desintegración social o la movilidad geográfica; por lo que consideramos prioritario poner especial atención a esta malla social, política, económica y cultural para sostener las relaciones interpersonales.

Interpelar la perspectiva desde la que nos posicionamos frente a las personas, cómo las miramos, es también una forma de construir. De alguna manera esta perspectiva de derechos nos habilita a pensar la situación de sufrimiento mental como una forma de manifestación del malestar social, para los cuales el tratamiento comunitario nos ofrece posesionarnos como profesionales desde una nueva perspectiva en ese campo. Entendemos que el sufrimiento está determinado por diversos factores que implican un "no acceso", reducción de las redes de apoyo, estar desvinculado de los capitales sociales, culturales, de los bienes y de los servicios ante una 
determinada situación. Por tanto, el entramado de redes permite repensar cuestiones multi-causales. Lo importante es entender desde dónde nos posicionamos frente al sufrimiento mental, lo que implicaría un posicionamiento ético-político.

Un análisis general de las entrevistas a los profesionales del Hospital T Vilardebó nos lleva a divisar que hay un discurso institucional y discursos profesionales muy internalizados que atraviesan las profesiones y las practicas empírico - profesionales; discursos permeados por una fuerte visión acerca de la división social del trabajo, muestra en psiquiatras una visión más pesimista de las redes al calificarlas de inexistentes o con fallas que no están siendo superadas, así como una visión más negativa de la familia (la cual a veces no entiende la enfermedad mental, desgastada, vulnerada, irresponsable). Los discursos de los trabajadores sociales y psicólogos coinciden en que las redes no son homogéneas ni democráticas en su acceso y valoran mucho el trabajo interdisciplinario e intersectorial.

Por otra parte, los licenciados en enfermería sostienen que es difícil de manejar la salida a la comunidad, y se muestran preocupados por cómo va a ser el seguimiento del tratamiento de las personas al egreso.

De manera general, interpretando los distintos discursos, vemos que es importante tener en cuenta que las redes que conforman el tejido social son promotoras de salud y bienestar y el principal sistema de apoyo natural al que acuden las personas; por lo que se deben diseñar y activar entramados de redes abiertas, múltiples que permitan tener un conocimiento del contexto del individuo para poder revertir situaciones de vulneraciones de derechos.
Cuando se trata de personas que han sufrido vulneraciones de derechos, se deben activar no solo redes de servicios de recursos sociales, sino de actividades recreativas, musicales, deportivas y de servicios territoriales, que permitan establecer entramados fuertes, abiertos, capaces de expandirse y multiplicarse ya que constituyen el principal sistema de apoyo natural al que las personas acuden en caso de necesidad. El pensar en redes es abandonar la postura inmediatista para la solución de problemas y asumir una perspectiva de participación más extensa e intensa a la vez, va a redundar en potenciar el apoyo social como determinante social de la salud indispensable para sostener en el afuera y revertir procesos de institucionalización psiquiátrica desde los derechos humanos. 


\section{Referencias bibliográficas}

1. Kessler RC, Price RH, Worthmann CB. Social factors in psychopathology. Annual Review of Psychology 1985; 36:531-72. Citado por: Villalba Quesada C. Redes sociales: un concepto con importantes implicaciones en la intervención comunitaria. [Internet]. Intervención psicosocial [Internet] 1993; 2(4). Disponible en: http://www. copmadrid.org/webcopm/publicaciones/ social/1993/vol1/arti6.htm [consulta: 31 jul 2019].

2. Salud Mental. Ley 19529 de 19 de septiembre de 2017. [Internet]. Uruguay. Disponible en: https://parlamento.gub.uy/ documentosyleyes/leyes?Ly_Nro=19529 [consulta: 31 jul. 2019].

3. Montero M. Teoría y práctica de la psicología comunitaria. Buenos Aires: Paidós; 2006. p. 173-98.

4. Berenstein I. Familia y enfermedad mental. Buenos Aires: Paidós; 1976. p. 75.

5. Bauman Z. La sociedad sitiada. Buenos Aires: Fondo de Cultura Económica; 2002. p. 115.

6. Levinas E. Totalidad e infinito: ensayo sobre la exterioridad. Salamanca: Sígueme; 2012.

7. Baremblit G. El movimiento instituyente, el autoanálisis y la autogestión. En: Baremblit G. Compendio de análisis institucional. Buenos Aires: Editorial Madres de Plaza de Mayo; 2005. p. 22.

8. Hopenhayn M. Inclusión y exclusión social en la juventud latinoamericana. [Internet]. 2008. p. 51. Disponible en: http:// dialnet.unirioja.es/servlet/articulo?codigo $=2781553$ [consulta: 31 jul 2019].
9. Naciones Unidas. La Declaración Universal de Derechos Humanos. [Internet]. 1948. Disponible en: https://www.un.org/ es/universal-declaration-human-rights/ [consulta: 31 jul. 2019].

\section{Bibliografía consultada}

Bourdieu P. Poder, derecho y clases sociales. Bilbao: Desclée de Brouwer; 2001.

Deleuze G, Guattari F. Rizoma: Introducción. Valencia: Pre-textos; 2003.

Frankel D. Medicalización de la vida. Buenos Aires: De la UNLa; 2008.

Foucault M. Los anormales. Buenos Aires: Fondo de Cultura Económica; 2000.

Foucault M. Las redes del poder. Buenos Aires: Prometeo; 2014.

Recibido: 20191029

Aceptado: 20191202 